\title{
Geographic and Ethnic Variations in Serum Concentrations of Legacy Persistent Organic Pollutants among Men in the Nenets Autonomous Okrug, Arctic Russia
}

\author{
Yulia Varakina ${ }^{1, *(D)}$, Andrey Aksenov ${ }^{1}$ (D), Dmitry Lakhmanov ${ }^{2}$, Anna Trofimova ${ }^{1}$ (D), Rimma Korobitsyna ${ }^{1}$ (D), \\ Natalia Belova ${ }^{1,3}$, Dmitry Kotsur ${ }^{1,4}$, Tatiana Sorokina ${ }^{1}{ }^{D}$, Andrej M. Grjibovski 1,3,5,6 ${ }^{\mathbb{D}}$, Ludmila Popova ${ }^{7}$, \\ Valery Chashchin ${ }^{1,8,9}$, Jon Øyvind Odland ${ }^{10,11}$ and Yngvar Thomassen 1,9,12 $\mathbb{D}$
}

check for updates

Citation: Varakina, Y.; Aksenov, A.; Lakhmanov, D.; Trofimova, A.; Korobitsyna, R.; Belova, N.; Kotsur, D.; Sorokina, T.; Grjibovski, A.M. Popova, L.; et al. Geographic and Ethnic Variations in Serum Concentrations of Legacy Persistent Organic Pollutants among Men in the Nenets Autonomous Okrug, Arctic Russia. Int. J. Environ. Res. Public Health 2022, 19, 1379. https:// doi.org/10.3390/ijerph19031379

Academic Editor: Paul B. Tchounwou

Received: 1 December 2021

Accepted: 25 January 2022

Published: 26 January 2022

Publisher's Note: MDPI stays neutral with regard to jurisdictional claims in published maps and institutional affiliations.

Copyright: (C) 2022 by the authors. Licensee MDPI, Basel, Switzerland. This article is an open access article distributed under the terms and conditions of the Creative Commons Attribution (CC BY) license (https:// creativecommons.org/licenses/by/ $4.0 /)$.
1 Arctic Biomonitoring Laboratory, Northern (Arctic) Federal University Named after M. V. Lomonosov, Naberezhnaya Severnoy Dvini 17, 163002 Arkhangelsk, Russia; a.s.aksenov@narfu.ru (A.A.); a.trofimova@narfu.ru (A.T.); r.korobicina@narfu.ru (R.K.); belova-8@mail.ru (N.B.); d.kocur@narfu.ru (D.K.); t.sorokina@narfu.ru (T.S.); andrej.grjibovski@gmail.com (A.M.G.); valerych05@mail.ru (V.C.); yngvar.thomassen@stami.no (Y.T.)

2 Laboratory of Environmental Analytical Chemistry, Core Facility Center "Arktika", Northern (Arctic) Federal University Named after M. V. Lomonosov, Naberezhnaya Severnoy Dvini 17, 163002 Arkhangelsk, Russia; d.lahmanov@narfu.ru

3 Northern State Medical University, Troitskiy Ave. 51, 163000 Arkhangelsk, Russia

4 N. Laverov Federal Center for Integrated Arctic Research, Ural Branch of the Russian Academy of Sciences, Naberezhnaya Severnoy Dvini 23, 163000 Arkhangelsk, Russia

5 Department of Epidemiology and Modern Vaccination Technology, I.M. Sechenov First Moscow State Medical University (Sechenov University), Trubetskaya Str., 8-2, 119991 Moscow, Russia

6 West Kazakhstan Marat Ospanov Medical University, Aktobe 0300190, Kazakhstan

7 Department of Chemistry and Chemical Ecology, Northern (Arctic) Federal University Named after M. V. Lomonosov, Naberezhnaya Severnoy Dvini 17, 163002 Arkhangelsk, Russia; lf.popova@narfu.ru

8 North-Western State Medical University Named after I. I. Mechnikov, Kirochnaya ul. 41, 191015 Saint-Petersburg, Russia

9 Institute of Ecology, National Research University Higher School of Economics, Myasnitskaya Str. 20, 101000 Moscow, Russia

10 Department of Public Health and Nursing, Norwegian University of Science and Technology, 7491 Trondheim, Norway; jon.o.odland@ntnu.no

11 Department of General Hygiene, I.M. Sechenov First Moscow State Medical University (Sechenov University), Trubetskaya Str., 8-2, 119992 Moscow, Russia

12 National Institute of Occupational Health, Gydas vei 8, N-0304 Oslo, Norway

* Correspondence: yu.varakina@narfu.ru; Tel.: +7-911-597-6935

Abstract: The overwhelming majority of Arctic biomonitoring studies in humans include either pregnant or non-pregnant women of reproductive age while little attention is paid to toxic compounds concentrations in men. This study contributes with information of the present amounts of persistent organic pollutants (POPs) in men living in Arctic Russia. We studied the serum concentrations of 11 polychlorinated biphenyl (PCB) congeners and 17 organochlorine pesticides (OCPs) and some of their metabolites in samples collected from 92 adult men (mean age 43 years) from seven different settlements in Nenets Autonomous Okrug (NAO). The median concentrations of individual PCB congeners increased in the order PCB 183, PCB 180, PCB 118, PCB 138, PCB 153. The concentrations of $\mathrm{o}, \mathrm{p}^{\prime}$-DDD, $\mathrm{p}, \mathrm{p}^{\prime}$-DDD, aldrin, mirex and 1,2,3,5-TCB were in most cases below the quantification limit. The observed concentrations of PCBs and chlorinated pesticides were in the same range as those found in similar groups of women of these territories, but lower than of men in other Arctic countries. However, significant geographic differences between the settlements were observed with exceptionally high concentrations of PCBs in the Islands group. The highest serum $\sum$ PCBs and $\beta-\mathrm{HCH}$ levels were observed in adult males aged 60-78 years. We found significant variations in serum concentrations of POPs across settlements and ethnic groups with exceptionally high concentrations of PCBs among the residents of the Arctic islands. At the same time, our findings suggest a considerable decrease in serum concentration of POPs over the last decade. 
Keywords: indigenous peoples; biomonitoring; Russian Arctic; men; PCBs; pesticides

\section{Introduction}

Persistent organic pollutants (POPs) are hazardous toxic substances with a high affinity for lipids and long half-lives [1]. Some of these compounds, for example $\mathrm{HCB}$ or $\mathrm{HCH}$, preferentially condense at temperatures about $-30^{\circ} \mathrm{C}$ [2].

POPs enter the Arctic ecosystem through atmospheric circulation, oceanic currents, river flow and biological pathways with the risk for negative consequences for human health and the environment [3].

Legacy POPs have been extensively monitored in the Arctic over the last decades, but most of the research have concentrated on the POPs contents in biological fluids of either pregnant or non-pregnant women of reproductive age [4-9]. However, studies from Greenland and Canada have presented data of POP levels in serum of men [10-12]. High levels of serum POPs such as p, p'-DDE and PCBs 118, 153,138,180 and 183 have been reported in men in [10], although the corresponding levels of serum POPs reported from the areas have been shown to be considerably lower than in, for example, northern Italy or southern Sweden $[13,14]$. A sufficient amount of evidence has been collected on deleterious effects of POPs on men's health $[2,9,13,15]$.

Our earlier research has demonstrated low levels of POPs in fish muscles and in the serum of women in the NAO, which is in line with the findings from other Arctic settings. Men living in Arctic Russia have significantly lower life expectancy, higher mortality and poorer general health compared to the national average [16]. Moreover, indigenous men in the Arctic have been reported to have poorer health and more health risks than indigenous women [17], warranting more research on both social and environmental determinants of health of men living in the Arctic.

Long-term exposures to organochlorine pollutants may cause gonadotoxic, embryotoxic and mutagenic effects in males [18]. For example, DDT increases the risk of male infertility [19] and PCBs cause inhibition of the production of sex hormones and deteriorate the quality of semen $[20,21]$. The most recent data on the concentration of POPs in serum of men was obtained from samples collected more than 10 years ago (2001-2010) in Chukotka in the north-east and in NAO in the north-west of Russia [22]. During that period, serum concentrations of DDT and p, $\mathrm{p}^{\prime}$-DDE decreased, while no changes have been observed for other POPs. The Arctic Biomonitoring Laboratory was established in 2017 to study effects of POPs in traditional food products on human health in Arctic Russia [23]. Our previous studies have indicated that the level of POPs in fish muscles [24] and in the serum of women [8] in NAO are low and similar to the findings from other Arctic countries.

However, the concentrations of legacy POPs in men in Arctic Russia have not been studied for more than a decade.

The aim of this paper was to present geographic and ethnic variations in serum concentrations of POPs among men permanently living in NAO, European Arctic Russia.

\section{Materials and Methods}

\subsection{Study Design}

All residents in seven settlements, namely, Bugrino, Varnek, Shoina, Indiga, Amderma, Krasnoe and Nelmin-Nos in NAO were invited to participate in the study. Detailed description of the settlements and recruitment procedures have been presented in our earlier publications $[8,25]$. Only men were selected for the purpose of this study $(n=92)$. Participants from the two island locations (Varnek, $n=6$ and Kolguev, $n=13$ ) were merged into one group titled the Islands, and participants from the two settlements located on the Pechora river bank (Nelmin-Nos, $\mathrm{n}=22$ and Krasnoe, $\mathrm{n}=8$ ) were merged into one group titled Pechora, considering similarities in environmental, social and dietary characteristics $[8,26]$ (Figure 1 ). 


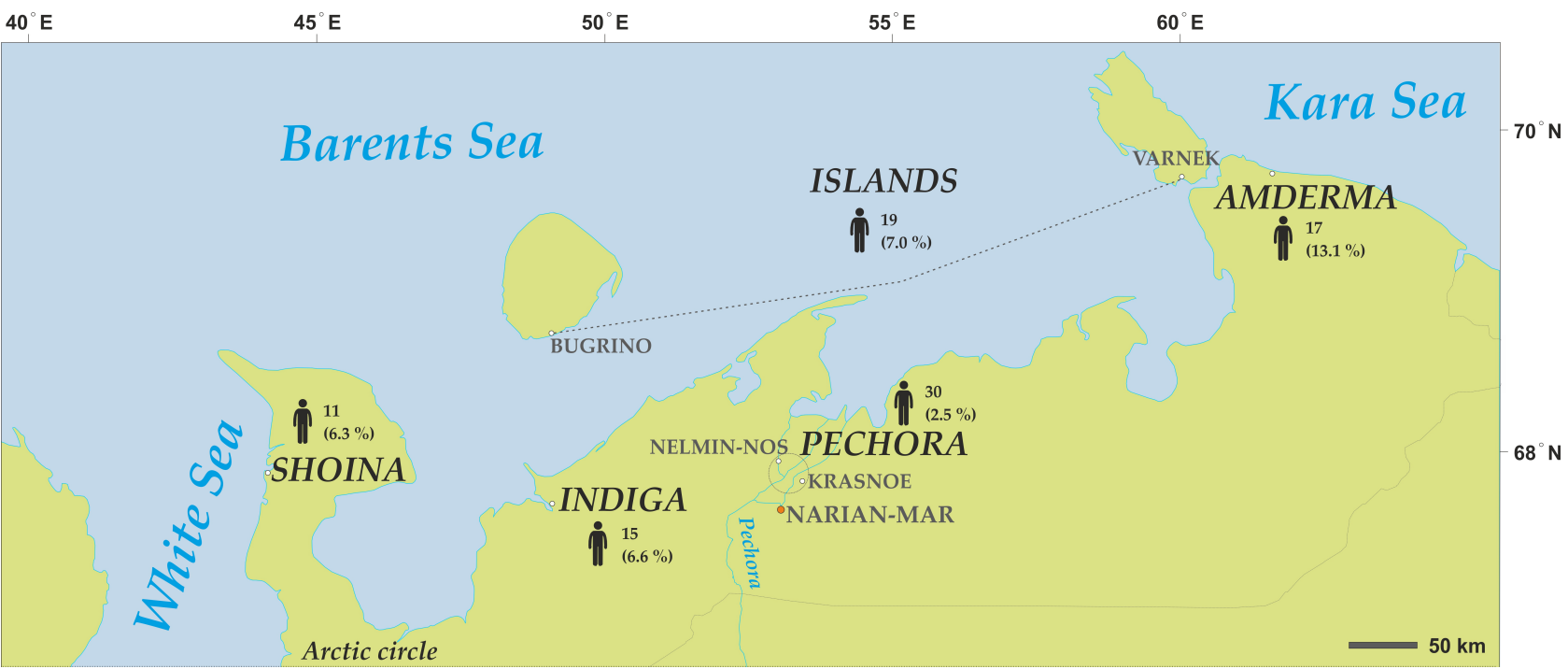

Figure 1. Location of the study settlements, number of study participants and proportion of the total male population. The map was created using CorelDRAW Graphics Suite X4 software (license certificate No 30064931). (https:/ / www.coreldraw.com/) (accessed on 20 July 2021); the topographic base of the map was created with Natural Earth Free Vector and Raster Map Data (https:/ / www. naturalearthdata.com) (accessed on 20 July 2021).

Ethnic background was registered as reported by the responders. Weight and height were measured, and body mass index (BMI) was calculated.

\subsection{Serum Samples}

A fasting blood sample from the cubital vein was taken from participants for POPs measurements: $p, p^{\prime}-D D E, p, p^{\prime}-D D D, o, p^{\prime}-D D E, o, p^{\prime}-D D D$, hexachlorobenzene (HCB), $\alpha$-hexachlorocyclohexane $(\alpha-\mathrm{HCH}), \beta$-hexachlorocyclohexane $(\beta-\mathrm{HCH}), \gamma$-hexachlorocyclohexane $(\gamma-\mathrm{HCH})$, heptachlor, cis-chlordane, trans-chlordane, cis-nonachlor, trans-nonachlor, aldrin, mirex, 1,2,3,5-tetrachlorobenzene (1,2,3,5-TCB) and 1,2,4,5-tetrachlorobenzene (1,2,4,5-TCB), as well as the polychlorinated biphenyls (PCBs): 28, 52, 101, 105, 118, 123, 128, 138, 153, 180, 183 .

For whole blood sampling we used $9 \mathrm{~mL}$ vacutainers Improvacuter (Guangzhou, China), Sarstedt pipettes (Nümbrecht, Germany) for further blood processing and vials (Glasstechnik Grafenroda, Geratal, Germany). A whole blood sample was taken from each examined person, from which a blood serum sample was obtained after centrifugation (3000 rpm). Serum was then transferred to $10 \mathrm{~mL}$ vials (Glasstechnik Grafenroda, Geratal, Germany) and frozen to $-25^{\circ} \mathrm{C}$ before transportation to $\mathrm{NaArFU}$ in medical cooler bags for long-term storage at $-25^{\circ} \mathrm{C}$ until analysis.

\subsection{Determination of Contaminants and Total Lipids}

The analysis of the following POPs set in analytes was carried out in the Core Facility Center "Arktika" of NArFU named after M.V. Lomonosov on a 7890 A gas chromatograph with an Agilent 7000 series triple quadrupole MS/MS system (Santa Clara, CA, USA) operating in the electron ionization mode $(70 \mathrm{eV})$ and with a capillary column HP-5MSUI (30 m $\times 0.25 \mathrm{~mm} \times 0.25 \mu \mathrm{m})$. Quality assurance and control of the determination of analytes in the sample was made by validation of each daily batch including blanks of hexane and matrix. The matrix blank was obtained by mixing equal parts of different serums. A detailed method and conditions for the determination of POPs are described elsewhere [8]. The yields of contaminants ranged from 86 to $120 \%$.

It should be noted that the limit of detection (LOD) and limit of quantification (LOQ) in $\mathrm{ng} / \mathrm{g}$ lipids is calculated taking into account the total lipid serum content which is different for each sample. The resulting LOD and LOQ ranges, depending on the minimum and 
maximum lipid levels, as well as the percentage of detection of analytes in the samples are presented in Table 1.

Table 1. Limit of detection (LOD), limit of quantification (LOQ) and the percentage of analytes detected in sample.

\begin{tabular}{|c|c|c|c|c|c|}
\hline \multirow{2}{*}{ Analyte } & \multirow{3}{*}{$\begin{array}{c}\%>\text { LOD }^{a} \\
-b\end{array}$} & \multirow{2}{*}{\multicolumn{2}{|c|}{$\begin{array}{c}\text { LOD } \\
\text { Range (ng/g Lipid) }\end{array}$}} & \multirow{2}{*}{\multicolumn{2}{|c|}{$\begin{array}{c}\text { LOQ } \\
\text { Range (ng/g Lipid) }\end{array}$}} \\
\hline & & & & & \\
\hline РCB28 & & 0.40 & 1.14 & 1.80 & 5.07 \\
\hline PCB52 & - & 2.92 & 8.24 & 9.74 & 27.5 \\
\hline РCB101 & - & 4.11 & 11.6 & 13.7 & 38.6 \\
\hline PCB105 & - & 5.92 & 16.7 & 19.7 & 55.6 \\
\hline PCB118 & 18.5 & 8.20 & 19.3 & 23.9 & 67.4 \\
\hline PCB123 & - & 4.43 & 12.5 & 14.8 & 41.6 \\
\hline PCB128 & - & 4.22 & 11.9 & 14.1 & 39.7 \\
\hline РCB138 & 42.4 & 9.50 & 22.4 & 27.8 & 78.4 \\
\hline PCB153 & 83.7 & 2.52 & 5.95 & 7.38 & 20.8 \\
\hline PCB180 & 58.7 & 1.23 & 2.90 & 3.59 & 10.1 \\
\hline PCB183 & 5.43 & 5.49 & 12.9 & 16.1 & 45.3 \\
\hline $\mathrm{o}, \mathrm{p}^{\prime}-\mathrm{DDE}$ & 82.6 & 2.06 & 4.86 & 6.03 & 17.0 \\
\hline $\mathrm{p}, \mathrm{p}^{\prime}-\mathrm{DDE}$ & 90.2 & 10.9 & 25.7 & 31.9 & 89.9 \\
\hline $0, p^{\prime}-D D D$ & 6.52 & 0.84 & 1.98 & 2.46 & 6.94 \\
\hline $\mathrm{p}, \mathrm{p}^{\prime}$-DDD & 34.8 & 4.78 & 11.3 & 14.0 & 39.5 \\
\hline $\mathrm{HCB}$ & 98.9 & 3.50 & 8.25 & 10.2 & 28.9 \\
\hline$\alpha-\mathrm{HCH}$ & - & 5.59 & 15.8 & 18.6 & 52.5 \\
\hline$\beta-\mathrm{HCH}$ & 96.7 & 2.00 & 4.71 & 5.84 & 16.5 \\
\hline$\gamma-\mathrm{HCH}$ & - & 3.62 & 10.2 & 12.1 & 34.0 \\
\hline cis-nonachlor & - & 3.51 & 9.91 & 11.7 & 33.0 \\
\hline trans-nonachlor & 8.70 & 8.85 & 20.9 & 25.9 & 73.0 \\
\hline cis-chlordane & - & 3.51 & 9.91 & 11.7 & 33.0 \\
\hline trans-chlordane & - & 8.56 & 24.1 & 28.5 & 80.4 \\
\hline Heptachlor & - & 7.68 & 21.7 & 25.6 & 72.2 \\
\hline Aldrin & 6.52 & 9.53 & 22.5 & 21.7 & 61.2 \\
\hline Mirex & 2.17 & 6.10 & 14.4 & 17.9 & 50.3 \\
\hline 1,2,3,5-tetrachlorobenzene & 22.8 & 1.09 & 2.58 & 3.20 & 9.02 \\
\hline $1,2,4,5$-tetrachlorobenzene & - & 0.96 & 2.71 & 3.20 & 9.02 \\
\hline
\end{tabular}

The concentrations of toxicants are presented as the sum of DDT and its metabolites $(\Sigma D D T)$, and the sum of indicator congeners of PCBs $\left(\Sigma \mathrm{PCB}_{5}\right)$, expressed in $\mathrm{ng} / \mathrm{g}$ lipids serum $[27,28]$. The total lipid content was assessed considering the quantitative content of cholesterol and triglycerides in blood serum using an automatic biochemical analyzer Random Access A-15 (Biosystems, Barcelona, Spain) at the Central Scientific Research Laboratory at the Northern State Medical University in Arkhangelsk.

\subsection{Statistical Analysis}

Distribution of numeric data was assessed using Shapiro-Wilk tests. Given that serum concentrations of all POPs were skewed to the right, the data were logarithmically transformed and presented as geometric means (GM), minimum and maximum values. All values below LOD were assumed to be equal to $\frac{1}{2}$ LOD. Bivariate associations between concentrations of POPs and place of residence were studied using Kruskal-Wallis tests or one way analysis of variance (ANOVA) depending on the distribution. Mann-Whitney tests and Student's unpaired $t$-tests were applied to assess ethnic differences.

Independent associations between location, ethnicity and serum concentrations of POPs were studied using multivariable linear regression models on logarithmically transformed data with adjustment for age and BMI. Men living along the Pechora River comprised the reference group. Assumptions of linearity, normal distribution of the residuals, and homoscedasticity were tested graphically. They held for o, p-DDE, p, $\mathrm{p}^{\prime}$-DDE, $\Sigma D D T$, 
PCB118, PCB138, PCB153, PCB180, $\Sigma$ PCB, HCB and $\beta-\mathrm{HCH}$, therefore the analysis was limited to these pollutants. Crude $\left(b_{c}\right)$ and adjusted $\left(b_{a}\right)$ regression coefficients with $95 \%$ confidence intervals (CI) were calculated. All analyses were performed using IBM SPSS software package, version 23.0 (IBM Corp., Armonk, NY, USA).

The study was approved by the local ethics committee at the Northern State Medical University (protocol no. 06/09-17 of 27 September 2017).

\section{Results}

\subsection{Study Population}

The age of the study participants ranged from 18 to 78 years with a mean of 43 years. The majority (55.4\%) of the 92 men were Nenets. The rest were Russians (36.9\%), Ukrainians $(3.3 \%)$, Komi (2.2\%), Mari (11\%) and Udmurts (1.1\%). Among them only Nenets were an indigenous group in NAO; therefore, we grouped participants into Nenets and non-Nenets as in our earlier study [17]. BMI of the participants varied from 19.4 to $40.0 \mathrm{~kg} / \mathrm{m}^{2}$, with an average of $26.7 \mathrm{~kg} / \mathrm{m}^{2}$. Study participants from different locations did not differ by any of the studied characteristics except the BMI (Table 2).

Table 2. Background characteristics of the study participants, Nenets Autonomous Okrug, Arctic Russia.

\begin{tabular}{|c|c|c|c|c|c|c|c|c|c|c|}
\hline Characteristic & Measures & Islands & Shoina & Amderma & Indiga & Pechora & $p$-Value ${ }^{\text {a }}$ & Nenets & Non-Nenets & $p$-Value ${ }^{\mathrm{b}}$ \\
\hline \multirow{4}{*}{ Age, years } & $\mathrm{N}$ & 19 & 11 & 17 & 15 & 30 & \multirow{4}{*}{0.502} & 51 & 41 & \multirow[b]{4}{*}{0.801} \\
\hline & GM & 37.5 & 44.1 & 45.0 & 36.9 & 40.9 & & 40.2 & 41.06 & \\
\hline & Min & 18.0 & 28.0 & 28.0 & 19.0 & 20.0 & & 20.0 & 18 & \\
\hline & Max & 78.0 & 63.0 & 72.0 & 59.0 & 70.0 & & 78.0 & 72 & \\
\hline \multirow[t]{2}{*}{ Total lipid, mg/dL } & GM & 578 & 599 & 627 & 560 & 595 & \multirow{3}{*}{0.615} & 588 & 597 & \\
\hline & Min & 395 & 459 & 468 & 414 & 417 & & 395 & 414 & \\
\hline & Max & 1110 & 859 & 977 & 821 & 874 & & 1110 & 977 & 0.485 \\
\hline \multirow[t]{3}{*}{ BMI, $\left(\mathrm{kg} / \mathrm{m}^{2}\right)$} & GM & 25.0 & 27.4 & 28.3 & 27.7 & 25.2 & \multirow{4}{*}{0.018} & 26.1 & 26.7 & \multirow{3}{*}{0.719} \\
\hline & Min & 19.8 & 20.5 & 19.7 & 19.4 & 20.8 & & 19.8 & 19.4 & \\
\hline & Max & 34.5 & 37.1 & 37.1 & 36.0 & 40.0 & & 37.1 & 40.0 & \\
\hline $\mathrm{BMI}>30, \mathrm{n}(\%)$ & & $2(10.5)$ & 2 (18.2) & $8(47.1)$ & $6(40)$ & $4(13.3)$ & & $10(20)$ & $12(29)$ & \\
\hline $\begin{array}{l}\text { Proportion of } \\
\text { Nenets, n (\%) }\end{array}$ & & $17(89.5)$ & $1(9.10)$ & $5(29.4)$ & $6(40.0)$ & $22(73.3)$ & & & & \\
\hline
\end{tabular}

${ }^{a} p$-value for difference between the settlements calculated using one-way ANOVA.; ${ }^{b} p$-value for difference between the settings calculated using Student $t$-test.

\subsection{Serum concentrations of Lipophilic POPS}

We detected quantifiable concentrations of 5 PCB congeners $(118,138,153,180,183)$, 10 chlorinated pesticides ( $\mathrm{HCB}, \beta-\mathrm{HCH}$, trans-nonachlor, aldrin, mirex and 1,2,3,5-TCB) and DDT metabolites (p, $\mathrm{p}^{\prime}$-DDE. $\mathrm{p}, \mathrm{p}^{\prime}$-DDD, o, $\mathrm{p}^{\prime}$-DDE, o, $\mathrm{p}^{\prime}$-DDD). Concentrations of $\alpha-\mathrm{HCH}, \gamma-\mathrm{HCH}$, heptachlor, cis-chlordane, trans-chlordane, cis-nonachlor, 1, 2, 4, 5-TCB and PCBs $(28,52,101,105,123,128)$ were below the limit of quantitative detection $(<\mathrm{LOD})$ (Table 1). GMs, minimum and maximum serum concentrations of POPs are presented in Table 3.

o, $\mathrm{p}^{\prime}$-DDE, $\mathrm{p}, \mathrm{p}^{\prime}$-DDE, HCB and $\beta-\mathrm{HCH}$ were detected in $82 \%$ or $99 \%$ of all samples with GM values of $12.6,68.3,61.2$ and $15.9 \mathrm{ng} / \mathrm{g}$ lipids, respectively. Of the PCBs, PCB 153 was present with highest concentration (22.7 $\mathrm{ng} / \mathrm{g}$ lipids) and detected in $83.7 \%$ of the samples. PCB 138 and PCB 180 were detected in $42.4 \%$ and 58.7\% of the samples with GM values of 16.9 and $5.82 \mathrm{ng} / \mathrm{g}$ lipids, respectively. All other compounds were detected above the LOD in between 2 and $34.8 \%$ of the samples.

All further measurements were performed only for POPs above the quantitative detection limit in all studied locations and for which all assumptions for multiple linear regression held. These substances were p, $\mathrm{p}^{\prime}$-DDE, $\sum D D T$, PCB 118, PCB 138, PCB 153, PCB 180, $\Sigma$ PCB, HCB and $\beta-\mathrm{HCH}$. 
Table 3. Serum concentrations of POPs (ng/g lipids) in men of the Nenets Autonomous Okrug, Arctic Russia.

\begin{tabular}{|c|c|c|c|c|c|c|c|c|c|c|c|}
\hline Analyte & Measures & Islands & Shoina & Amderma & Indiga & Pechora & $p$-Value $^{3}$ & Total & Nenets & Non-Nenets & $p$-Value ${ }^{4}$ \\
\hline \multirow{2}{*}{\multicolumn{12}{|c|}{$\begin{array}{c}15 \\
\text { PCBs, ng/g lipid }\end{array}$}} \\
\hline & & & & & & & & & & & \\
\hline \multirow[t]{3}{*}{ PCB 118} & GM & 8.24 & $<$ LOD & 14.7 & 12.3 & $<\mathrm{LOD}$ & & 9.08 & $<\mathrm{LOD}$ & 10.5 & \\
\hline & Min & $<\mathrm{LOD}^{5}$ & $<$ LOD & $<\mathrm{LOD}$ & $<\mathrm{LOD}$ & $<$ LOD & $<0.001$ & $<\mathrm{LOD}$ & $<\mathrm{LOD}$ & $<\mathrm{LOD}$ & 0.049 \\
\hline & Max & 45.0 & 24.0 & 42.1 & 89.0 & $<\mathrm{LOD}$ & & 89.0 & 45.0 & 89.0 & \\
\hline \multirow{3}{*}{ РCB 138} & GM & 37.7 & 30.7 & 19.1 & 12.8 & $<\mathrm{LOD}$ & & 16.9 & 16.3 & 17.7 & \\
\hline & Min & $<$ LOD & $<$ LOD & $<\mathrm{LOD}$ & $<\mathrm{LOD}$ & $<\mathrm{LOD}$ & $<0.001$ & $<\mathrm{LOD}$ & $<\mathrm{LOD}$ & $<\mathrm{LOD}$ & 0.489 \\
\hline & Max & 515 & 96.8 & 60.0 & 163 & 54.6 & & 515 & 515 & 163 & \\
\hline \multirow[t]{3}{*}{ РCB 153} & GM & 90.4 & 41.6 & 31.5 & 12.7 & 8.34 & & 22.7 & 27.5 & 17.8 & \\
\hline & Min & $<\mathrm{LOD}$ & 8.84 & $<\mathrm{LOD}$ & $<\mathrm{LOD}$ & $<\mathrm{LOD}$ & $<0.001$ & $<\mathrm{LOD}$ & $<\mathrm{LOD}$ & $<\mathrm{LOD}$ & 0.156 \\
\hline & Max & 2640 & 142 & 83.3 & 292 & 77.2 & & 2640 & 2640 & 292 & \\
\hline \multirow[t]{3}{*}{ РCB 180} & GM & 52.9 & 11.9 & 15.7 & 3.32 & $<\mathrm{LOD}$ & & 5.82 & 5.84 & 5.80 & \\
\hline & Min & 6.55 & 2.22 & $<\mathrm{LOD}$ & $<\mathrm{LOD}$ & $<$ LOD & $<0.001$ & $<\mathrm{LOD}$ & $<$ LOD & $<\mathrm{LOD}$ & 0.102 \\
\hline & Max & 1420 & 37.4 & 52.4 & 118 & $<\mathrm{LOD}$ & & 1420 & 1420 & 118 & \\
\hline \multirow{3}{*}{ PCB 183} & GM & 6.84 & $<\mathrm{LOD}$ & $<\mathrm{LOD}$ & $<\mathrm{LOD}$ & $<\mathrm{LOD}$ & & $<\mathrm{LOD}$ & $<\mathrm{LOD}$ & $<\mathrm{LOD}$ & \\
\hline & Min & $<\mathrm{LOD}$ & $<\mathrm{LOD}$ & $<\mathrm{LOD}$ & $<\mathrm{LOD}$ & $<$ LOD & - & $<\mathrm{LOD}$ & $<\mathrm{LOD}$ & $<\mathrm{LOD}$ & 0.064 \\
\hline & Max & 45.6 & $<\mathrm{LOD}$ & $<\mathrm{LOD}$ & $<\mathrm{LOD}$ & $<$ LOD & & 45.6 & 45.6 & 6.03 & \\
\hline \multirow[t]{3}{*}{$\sum \mathrm{PCB}_{5}{ }^{1}$} & GM & 222 & 110 & 92.2 & 52.5 & 33.6 & & 74.1 & 80.2 & 67.2 & \\
\hline & Min & 28.8 & 35.1 & 20.6 & 18.9 & 15.0 & $<0.001$ & 15.0 & 19.3 & 15.0 & 0.165 \\
\hline & Max & 4600 & 257 & 217 & 666 & 137 & & 4600 & 4600 & 666 & \\
\hline \multicolumn{12}{|c|}{ OCPs ng/g lipid } \\
\hline \multirow[t]{3}{*}{$\mathrm{o}, \mathrm{p}-\mathrm{DDE}$} & GM & 21.7 & 23.0 & 6.57 & 3.81 & 18.6 & & 12.6 & 13.0 & 12.0 & \\
\hline & Min & 2.70 & 11.8 & $<\mathrm{LOD}$ & $<\mathrm{LOD}$ & $<\mathrm{LOD}$ & $<0.001$ & $<\mathrm{LOD}$ & $<\mathrm{LOD}$ & $<\mathrm{LOD}$ & 0.407 \\
\hline & Max & 61.8 & 38.5 & 23.7 & 24.0 & 75.2 & & 75.2 & 65.6 & 75.2 & \\
\hline \multirow{3}{*}{$\mathrm{p}, \mathrm{p}^{\prime}-\mathrm{DDE}$} & GM & 121 & 152 & 123 & 41.8 & 32.5 & & 68.3 & 48.7 & 104 & \\
\hline & Min & 30.6 & 43.3 & 22.7 & $<\mathrm{LOD}$ & $<$ LOD & $<0.001$ & $<\mathrm{LOD}$ & $<\mathrm{LOD}$ & 16.0 & 0.009 \\
\hline & Max & 467 & 549 & 1320 & 336 & 278 & & 1320 & 467 & 1320 & \\
\hline \multirow[t]{3}{*}{$\mathrm{o}, \mathrm{p}$-DDD } & GM & $<\mathrm{LOD}$ & $<$ LOD & $<$ LOD & $<\mathrm{LOD}$ & 0.99 & & 0.92 & 0.86 & 1.00 & \\
\hline & Min & $<\mathrm{LOD}$ & $<\mathrm{LOD}$ & $<\mathrm{LOD}$ & $<\mathrm{LOD}$ & $<\mathrm{LOD}$ & - & $<\mathrm{LOD}$ & $<\mathrm{LOD}$ & $<\mathrm{LOD}$ & 0.262 \\
\hline & Max & $<\mathrm{LOD}$ & $<\mathrm{LOD}$ & $<\mathrm{LOD}$ & $<\mathrm{LOD}$ & 129 & & 129 & 1.73 & 129 & \\
\hline \multirow[t]{3}{*}{$\mathrm{p}, \mathrm{p}^{\prime}-\mathrm{DDD}$} & GM & $<\mathrm{LOD}$ & $<\mathrm{LOD}$ & $<\mathrm{LOD}$ & $<\mathrm{LOD}$ & 47.5 & & 9.62 & 12.1 & 7.22 & \\
\hline & Min & $<\mathrm{LOD}$ & $<\mathrm{LOD}$ & $<\mathrm{LOD}$ & $<\mathrm{LOD}$ & 17.3 & & $<\mathrm{LOD}$ & $<\mathrm{LOD}$ & $<\mathrm{LOD}$ & 0.165 \\
\hline & Max & $<\mathrm{LOD}$ & $<\mathrm{LOD}$ & 8.41 & 6.03 & 183 & & 183 & 183 & 109 & \\
\hline \multirow[t]{3}{*}{$\sum \mathrm{DDT}^{2}$} & GM & 161 & 193 & 146 & 55.0 & 124 & & 125 & 109 & 146 & \\
\hline & Min & 61.3 & 72.4 & 41.1 & 15.3 & 36.2 & 0.002 & 15.3 & 15 & 21.4 & 0.019 \\
\hline & Max & 506 & 570 & 1321 & 342 & 534 & & 1320 & 506 & 1321 & \\
\hline \multirow{3}{*}{ НСВ } & GM & 83.1 & 26.3 & 34.2 & 133 & 65.1 & & 61.2 & 81.7 & 42.8 & \\
\hline & Min & $<\mathrm{LOD}$ & 10.85 & 9.4 & 22.2 & 12.5 & $<0.001$ & $<\mathrm{LOD}$ & $<\mathrm{LOD}$ & 9.38 & 0.014 \\
\hline & Max & 358 & 109 & 250 & 469 & 713 & & 713 & 713 & 370 & \\
\hline \multirow[t]{3}{*}{$\beta-\mathrm{HCH}$} & GM & 13.2 & 26.4 & 17.1 & 19.8 & 12.7 & & 15.9 & 13.2 & 19.9 & \\
\hline & Min & 2.75 & 3.17 & 4.00 & 7.21 & 2.10 & 0.227 & 2.10 & 2.75 & 2.10 & 0.006 \\
\hline & Max & 31.5 & 178 & 118 & 139 & 89.2 & & 178 & 139 & 178 & \\
\hline \multirow[t]{3}{*}{ Aldrin } & GM & 18.1 & $<\mathrm{LOD}$ & $<\mathrm{LOD}$ & $<\mathrm{LOD}$ & $<$ LOD & & $<\mathrm{LOD}$ & 10.5 & $<$ LOD & \\
\hline & Min & $<\mathrm{LOD}$ & $<\mathrm{LOD}$ & $<\mathrm{LOD}$ & $<\mathrm{LOD}$ & $<$ LOD & - & $<\mathrm{LOD}$ & $<\mathrm{LOD}$ & $<$ LOD & 0.138 \\
\hline & Max & 987 & $<\mathrm{LOD}$ & 24.6 & $<\mathrm{LOD}$ & $<$ LOD & & 987 & 987 & 24.6 & \\
\hline Mirex & GM & $<\mathrm{LOD}$ & $<\mathrm{LOD}$ & $<\mathrm{LOD}$ & $<\mathrm{LOD}$ & $<\mathrm{LOD}$ & & $<\mathrm{LOD}$ & $<\mathrm{LOD}$ & $<\mathrm{LOD}$ & \\
\hline & Min & $<\mathrm{LOD}$ & $<\mathrm{LOD}$ & $<\mathrm{LOD}$ & $<\mathrm{LOD}$ & $<$ LOD & & $<\mathrm{LOD}$ & $<\mathrm{LOD}$ & $<\mathrm{LOD}$ & 0.207 \\
\hline & Max & 36.6 & $<\mathrm{LOD}$ & $<\mathrm{LOD}$ & $<\mathrm{LOD}$ & $<\mathrm{LOD}$ & - & 36.6 & 36.6 & 7.24 & \\
\hline $\begin{array}{c}\text { trans- } \\
\text { nonachlor }\end{array}$ & GM & 11.9 & 9.60 & $<\mathrm{LOD}$ & $<\mathrm{LOD}$ & $<$ LOD & & $<\mathrm{LOD}$ & 9.05 & $<\mathrm{LOD}$ & \\
\hline & Min & $<\mathrm{LOD}$ & $<\mathrm{LOD}$ & $<\mathrm{LOD}$ & $<\mathrm{LOD}$ & $<$ LOD & - & $<\mathrm{LOD}$ & $<$ LOD & $<\mathrm{LOD}$ & 0.207 \\
\hline & Max & 95.7 & 34.8 & $<$ LOD & 26.1 & $<$ LOD & & 95.7 & 95.7 & 34.8 & \\
\hline $1,2,3,5-\mathrm{TCB}$ & GM & $<\mathrm{LOD}$ & $<\mathrm{LOD}$ & 1.73 & 64.9 & $<\mathrm{LOD}$ & & 1.96 & 1.52 & 2.70 & \\
\hline & Min & $<\mathrm{LOD}$ & $<\mathrm{LOD}$ & $<\mathrm{LOD}$ & 12.5 & $<$ LOD & - & $<\mathrm{LOD}$ & $<\mathrm{LOD}$ & $<\mathrm{LOD}$ & 0.798 \\
\hline & Max & $<\mathrm{LOD}$ & $<\mathrm{LOD}$ & 10.4 & 502 & $<\mathrm{LOD}$ & & 502 & 502 & 250 & \\
\hline
\end{tabular}

\subsection{Geographic Variations in Serum Concentration of POPs}

3.3.1. Crude Analysis

Significant variations in the concentrations of $\mathrm{p}, \mathrm{p}^{\prime}$-DDE, PCB 118, PCB 138, PCB 153, PCB 180, PCB, and HCB were observed between the settlements (Table 4). Concentrations of p, $\mathrm{p}^{\prime}$-DDE, PCB 138, PCB 153, $\mathrm{PCB}$ in the Islands, in Shoina and Amderma were 
significantly higher than in Pechora. The PCB 118 concentration in Amderma and Indiga was greater compared to the reference group. Concentration of PCB 180 in all settlements exceeded the levels in Pechora. HCB concentration in Pechora was greater than in Shoina and Amderma. At the same time, men living along the Pechora River had lower $\beta-\mathrm{HCH}$ levels than men living in Shoina. Detailed data on crude variations in serum concentration of selected POPs is presented in Table 4.

Table 4. Crude coefficients $\left(b_{c}\right)$ and $95 \%$ confidence intervals $(C I)$ for the associations between logarithmically transformed serum concentrations of legacy POPs and selected covariates among men in the Nenets Autonomous Okrug.

\begin{tabular}{|c|c|c|c|c|c|c|c|c|c|c|c|c|c|c|}
\hline \multirow[t]{3}{*}{ Variable } & \multicolumn{8}{|c|}{$\begin{array}{l}\text { Crude Differences between Pechora and Other } \\
\text { Locations }\end{array}$} & \multicolumn{2}{|c|}{ Crude Ethnic Differences } & \multicolumn{2}{|r|}{ Age } & \multicolumn{2}{|c|}{ BMI } \\
\hline & \multicolumn{2}{|c|}{ Islands } & \multicolumn{2}{|r|}{ Shoina } & \multicolumn{2}{|c|}{ Amderma } & \multicolumn{2}{|r|}{ Indiga } & \multicolumn{2}{|c|}{ Nenets vs. non-Nenets } & \multirow[b]{2}{*}{$\mathbf{b}_{\mathbf{c}}$} & \multirow[b]{2}{*}{$95 \%$ CI } & \multirow[b]{2}{*}{ B * } & \multirow[b]{2}{*}{$95 \%$ CI } \\
\hline & $b_{c} *$ & $95 \%$ CI & $\mathbf{b}_{\mathbf{c}}$ & $95 \%$ CI & $b_{c}$ & $95 \%$ CI & $\mathbf{b}_{\mathbf{c}}$ & $95 \%$ CI & $\mathbf{b}_{\mathbf{c}}$ & $95 \%$ CI & & & & \\
\hline $\mathrm{p}, \mathrm{p}^{\prime}-\mathrm{DDE}$ & 1.31 & $0.66 ; 1.96$ & 1.55 & $0.76 ; 2.32$ & 1.33 & $0.66 ; 2.01$ & 0.25 & $-0.45 ; 0.95$ & -0.76 & $-1.27 ;-0.25$ & 0 & $-0.02 ; 0.02$ & 0.07 & $0.01 ; 0.13$ \\
\hline$\sum \mathrm{DDT}$ & 0.26 & $-0.16 ; 0.69$ & 0.44 & $-0.07 ; 0.95$ & 0.16 & $-0.28 ; 0.60$ & -0.81 & $-1.27 ;-0.36$ & -0.29 & $-0.63 ; 0.04$ & 0.01 & $0.00 ; 0.02$ & 0.03 & $-0.01 ; 0.06$ \\
\hline PCB 118 & 0.20 & $-0.12 ; 0.53$ & 0.13 & $-0.26 ; 0.52$ & 0.78 & $0.44 ; 1.12$ & 0.61 & $0.26 ; 0.96$ & -0.26 & $-0.51 ;-0.01$ & 0.01 & $0.00 ; 0.02$ & 0.04 & $0.01 ; 0.08$ \\
\hline PCB 138 & 1.46 & $0.96 ; 1.96$ & 1.26 & $0.66 ; 1.85$ & 0.78 & $0.27 ; 1.30$ & 0.38 & $-0.16 ; 0.91$ & -0.08 & $-0.50 ; 0.34$ & 0.02 & $0.01 ; 0.03$ & 0.04 & $-0.01 ; 0.07$ \\
\hline PCB 153 & 2.38 & $1.64 ; 3.13$ & 1.61 & $0.71 ; 2.51$ & 1.33 & $0.56 ; 2.10$ & 0.42 & $-0.38 ; 1.23$ & 0.43 & $-0.21 ; 1.08$ & 0.03 & $0.01 ; 0.05$ & 0.01 & $-0.06 ; 0.09$ \\
\hline PCB 180 & 4.14 & $3.50 ; 4.79$ & 2.65 & $1.87 ; 3.43$ & 2.93 & $2.26 ; 3.60$ & 1.37 & $0.68 ; 2.07$ & 0.01 & $-0.80 ; 0.82$ & 0.02 & $-0.01 ; 0.05$ & 0.03 & $-0.06 ; 0.12$ \\
\hline$\sum \mathrm{PCB}$ & 1.89 & $1.39 ; 2.38$ & 1.19 & $0.59 ; 1.78$ & 1.01 & $0.50 ; 1.52$ & 0.45 & $-0.09 ; 0.98$ & 0.18 & $-0.28 ; 0.63$ & 0.02 & $0.01 ; 0.03$ & 0.02 & $-0.03 ; 0.08$ \\
\hline $\mathrm{HCB}$ & 0.25 & $-0.28 ; 0.77$ & -0.91 & $-1.53 ;-0.28$ & -0.64 & $-1.18 ;-0.11$ & 0.71 & $0.15 ; 1.27$ & 0.65 & $0.24 ; 1.05$ & 0.01 & $-0.01 ; 0.02$ & 0.02 & $-0.03 ; 0.06$ \\
\hline$\beta-\mathrm{HCH}$ & 0.04 & $-0.46 ; 0.54$ & 0.73 & $0.13 ; 1.33$ & 0.30 & $-0.22 ; 0.81$ & 0.44 & $-0.10 ; 0.98$ & -0.41 & $-0.76 ;-0.05$ & 0.02 & $0.01 ; 0.03$ & 0.06 & $0.02 ; 0.10$ \\
\hline
\end{tabular}

* Statistically significant associations are in bold.

\subsubsection{Adjusted Analysis}

Adjustment for age, BMI and ethnic background attenuated the differences in $\mathrm{p}, \mathrm{p}^{\prime}$-DD concentrations between Amderma and Pechora, but mainly between the Islands and the reference group. Geographic variations in serum concentrations of PCB 118, PCB 138, 153, PCB 180, $\sum$ PCB and HCB remained significant while no independent associations between location and $\beta-\mathrm{HCH}$ were observed in multivariable analysis (Table 5).

Table 5. Adjusted coefficients $\left(b_{a}\right)$ and $95 \%$ confidence intervals $(C I)$ for the associations between logarithmically transformed serum concentrations of legacy POPs and selected covariates among men in the Nenets Autonomous Okrug.

\begin{tabular}{|c|c|c|c|c|c|c|c|c|c|c|c|c|c|c|}
\hline \multirow[t]{3}{*}{ Variable } & \multicolumn{8}{|c|}{$\begin{array}{l}\text { Adjusted Differences between Pechora and Other } \\
\text { Locations }\end{array}$} & \multirow{2}{*}{\multicolumn{2}{|c|}{$\begin{array}{c}\text { Adjusted Ethnic Differences } \\
\text { Nenets vs. Non-Nenets }\end{array}$}} & \multicolumn{2}{|r|}{ Age } & \multicolumn{2}{|r|}{ BMI } \\
\hline & \multicolumn{2}{|c|}{ Islands } & \multicolumn{2}{|r|}{ Shoina } & \multicolumn{2}{|c|}{ Amderma } & \multicolumn{2}{|r|}{ Indiga } & & & & & & \\
\hline & $\mathrm{b}_{\mathrm{a}}$ * & $95 \% \mathrm{CI}$ & $\mathbf{b}_{\mathbf{a}}{ }^{*}$ & $95 \%$ CI & $\mathbf{b}_{\mathbf{a}} *$ & $95 \%$ CI & $\mathbf{b}_{\mathbf{a}}$ * & $95 \%$ CI & $\mathbf{b}_{\mathbf{a}}$ * & $95 \% \mathrm{CI}$ & $\mathbf{b}_{\mathbf{a}}{ }^{*}$ & $95 \%$ CI & $\mathbf{b}_{\mathbf{a}}{ }^{*}$ & $95 \%$ CI \\
\hline $\mathrm{p}, \mathrm{p}^{\prime}-\mathrm{DDE}$ & 1.38 & $0.75 ; 2.02$ & 0.91 & $0.08 ; 1.73$ & 0.62 & $-0.10 ; 1.43$ & -0.30 & $-1.01 ; 0.41$ & -0.80 & $-1.32 ;-0.27$ & 0.00 & $-0.02 ; 0.01$ & 0.07 & $0.02 ; 0.13$ \\
\hline$\sum$ DDT & 0.33 & $-0.08 ; 0.74$ & 0.01 & $-0.52 ; 0.54$ & -0.32 & $-0.78 ; 0.15$ & -1.13 & $-1.59 ;-0.68$ & -0.51 & $-0.85 ;-0.17$ & 0.01 & $-0.01 ; 0.02$ & 0.04 & $0.01 ; 0.08$ \\
\hline PCB 118 & 0.23 & $-0.11 ; 0.56$ & 0 & $-0.44 ; 0.43$ & 0.60 & $0.22 ; 0.98$ & 0.50 & $0.12 ; 0.88$ & 0.13 & $-0.41 ; 0.15$ & 0.01 & $0.00 ; 0.02$ & 0.02 & $-0.01 ; 0.05$ \\
\hline PCB 138 & 1.53 & $1.04 ; 2.01$ & 1.13 & $0.50 ; 1.76$ & 0.56 & $0.01 ; 1.11$ & 0.33 & $-0.22 ; 0.87$ & -0.07 & $-0.48 ; 0.33$ & 0.02 & $0.01 ; 0.03$ & 0.03 & $-0.01 ; 0.07$ \\
\hline PCB 153 & 2.33 & $1.60 ; 3.06$ & 1.82 & $0.89 ; 2.78$ & 1.47 & $0.64 ; 2.29$ & 0.70 & $-0.12 ; 1.52$ & 0.54 & $-0.06 ; 1.15$ & 0.03 & $0.01 ; 0.04$ & 0.00 & $-0.06 ; 0.06$ \\
\hline PCB 180 & 4.24 & $3.58 ; 4.90$ & 2.70 & $1.85 ; 3.56$ & 2.95 & $2.21 ; 3.70$ & 1.48 & $0.74 ; 2.22$ & 0.17 & $-0.38 ; 0.72$ & 0.02 & $0.00 ; 0.04$ & 0.00 & $-0.06 ; 0.05$ \\
\hline$\sum$ PCB & $\begin{array}{l}4.24 \\
1.90\end{array}$ & $\begin{array}{l}3.30 ; .90 \\
1.42 ; 2.38\end{array}$ & 1.19 & $\begin{array}{l}1.05 ; 3.006 \\
0.57 ; 1.82\end{array}$ & 0.94 & $0.40 ; 1.49$ & $\begin{array}{l}1.48 \\
0.52\end{array}$ & $\begin{array}{c}0.14 ; 2.22 \\
-0.03 ; 1.01\end{array}$ & 0.17 & $\begin{array}{l}-0.00,0.72 \\
-0.23 ; 0.57\end{array}$ & $\begin{array}{l}.02 \\
0.02\end{array}$ & $\begin{array}{l}0.00 ; ; 0.04 \\
0.01 ; 0.03\end{array}$ & 0.01 & $\begin{array}{l}-0.03 ; 0.05 \\
-0.03 ; 0.05\end{array}$ \\
\hline $\mathrm{HCB}$ & 0.20 & $-0.32 ; 0.73$ & -0.73 & $-1.41 ;-0.05$ & -0.66 & $-1.25 ;-0.07$ & 0.74 & $0.15 ; 1.33$ & 0.42 & $-0.02 ; 0.86$ & 0.01 & $0.00 ; 0.02$ & 0.02 & $-0.02 ; 0.07$ \\
\hline$\beta-\mathrm{HCH}$ & 0.06 & $-0.40 ; 0.52$ & 0.39 & $\begin{array}{l}-0.21 ; 0.99 \\
-1.45\end{array}$ & $\begin{array}{l}-0.00 \\
-0.15\end{array}$ & $-0.67 ; 0.37$ & 0.25 & $-0.27 ; 0.76$ & $\begin{array}{l}-0.42 \\
-0.30\end{array}$ & $\begin{array}{l}-0.02 ; 0.00 \\
-0.68\end{array}$ & 0.02 & $0.01 ; 0.03$ & 0.05 & $0.01 ; 0.09$ \\
\hline
\end{tabular}

* Adjusted for age, body mass index, ethnic background and location. Statistically significant associations are in bold.

\subsection{Ethnic Variations in Serum Concentration of POPS}

\subsubsection{Crude Analysis}

We observed ethnic differences in serum concentration of $p, p^{\prime}$-DDE, PCB 118 and HCB (Table 4). Nenets men had higher concentration of HCB, but lower levels of $p, p^{\prime}-D D E$ and PCB 118.

\subsubsection{Adjusted Analysis}

Only $\mathrm{p}, \mathrm{p}^{\prime}$-DDE and $\sum$ DDT concentrations remained significantly lower in the Nenets compared to non-Nenets. All other associations decreased to non-significant levels.

Age was significantly associated with PCB 138, PCB 153, PCB 180, $\sum$ PCB and $\beta-H C H$ while BMI was associated with $\mathrm{p}, \mathrm{p}^{\prime}-\mathrm{DDE}, \sum \mathrm{DDT}$ and $\beta-\mathrm{HCH}$ in multivariable analysis (Table 5). 


\section{Discussion}

To our knowledge, this is the first study of the distribution of POPs in men across settlements and ethnic groups in Arctic Russia.

Although recent data on POPs from Russia are available in international literature, these studies included only women [7,8,29]. Studies from Greenland and Canada have reported concentrations of POPs by gender [10-12] while the data from NAO [3,30] have not been stratified. Therefore, our results can be used as a starting point for monitoring of POPs in men in European Arctic Russia.

The most detected pesticide in the men's serum in Arctic Russia is p, p'-DDE, which is similar to the results from the United States, Europe, and China [31]. Geometric mean values for $\sum \mathrm{PCB}_{5}$ and DDT in men's serum were 74.1 and $125 \mathrm{ng} / \mathrm{g}$ lipids, respectively (Table 3), which is from 6 to 11 times as low as reported in earlier publications from northwestern Russia [32]. Concentrations of $\Sigma \mathrm{PCB}_{5}$ among Inuits in Canada $[10,11]$ were four times as high as in our study. Residents of Greenland had 40 times as high concentrations of PCBs [12] as the residents of NAO. High levels of PCBs are likely to be associated with the more frequent consumption of sea animals [33,34] in Greenland and Arctic Canada than in NAO.

The only settlement with available serum concentrations of selected POPs from earlier periods was Nelmin-Nos. Our results from the same age group suggest a decrease in the serum concentrations of $p, p^{\prime}$-DDE, HCB, PCBs $(153,180,138,118)$ compared to the results from 2009 ([32], Figure 2).

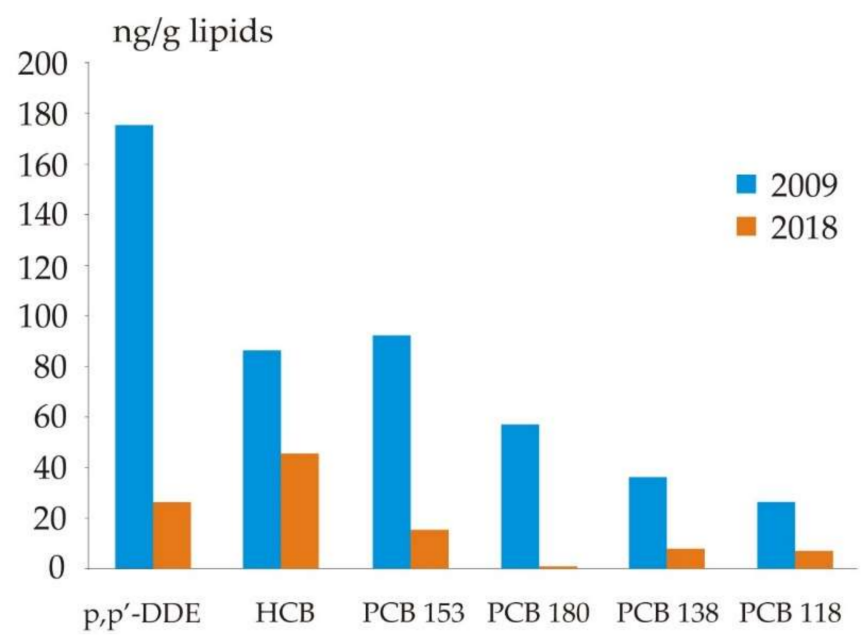

Figure 2. Median serum concentration of individual POPs in men in 2009 [32] and 2018 in NelminNos, Nenets Autonomous Okrug, Arctic Russia.

A decrease in serum concentrations of POPs has also been observed in men in other regions. For example, concentrations of PCB 153 and p, p'-DDE in Greenland decreased annually by $6.67-8.61 \%$ and by $6.11-9.52 \%$, respectively, between 1997 and 2007 [33]. In Barcelona, the concentrations of PCB 153, p, $\mathrm{p}^{\prime}$-DDE, HCB and $\beta-\mathrm{HCH}$ decreased by $10 \%-75 \%$ between 2006 and 2016 [34]. This is most likely a result of adoption of the Stockholm Convention on Persistent Organic Pollutants in 2004 aiming at elimination or limiting the production and use of POPs [9]. Replacement of traditional diet by processed foods including imported food items may also have contributed to the observed trend in POPs concentrations in Arctic Russia.

The main factors associated with the accumulation of POPs are age, BMI, environment and nutrition [14,35-39]. In addition to these main factors, there are ethnic [25] and geographical $[33,40]$ variations. For example, the highest concentrations of $p, p^{\prime}-D D E$, PCB 153 were observed in the north of Greenland compared to other parts of the island [40].

Similarly, residents of the Islands and Shoina located in the north and northwest of NAO had higher serum concentrations of $\mathrm{p}, \mathrm{p}^{\prime}-\mathrm{DDE}$, which is the most persistent 
metabolite of DDT [41]. Coastal areas and the islands of NAO were most likely to be contaminated by long-range transport of DDT prior to mitigation measures under the Stockholm Convention. On the other hand, there is a local pollution of the environment with these compounds $[38,39]$, resulting in human body contamination through the local food chains [42-44].

High levels of PCBs (PCB 138, PCB 153, PCB 180, $\sum$ PCB) are common in all areas of NAO except Indiga and Pechora. Men living on the islands have higher concentration of PCB 138, PCB 153, PCB 180 than men living in other settlements. This is in line with the findings on serum concentrations of POPs among women from the same settlements [8]. Higher concentrations of PCBs in both men and women living on the islands may be at least partly attributed to consumption of sea mammals [45], which have been shown to have concentrations of PCBs more than 10 times as high as other traditional foods in the Arctic $[4,24,40,46]$. More research is needed to explain the nutritional factors behind the observed differences in serum concentrations of POPs among the residents of the Arctic islands. Residents of Shoina and Amderma have lower serum levels of than those of Pechora and Indiga. Freshwater fish and dairy products have been previously shown to be associated with HCB concentrations $[47,48]$. According to the food frequency questionnaire [49], residents of Pechora more frequently consume dairy products, mainly from the dairy factory located nearby.

Ethnic variations in serum concentrations of selected POPs have also been observed in other Arctic settings, potentially reflecting the differences in traditional nutrition. For example, indigenous people in Greenland had higher concentrations of PCB and HCB [12], which was explained by nutritional factors. Indigenous people are more likely to consume traditional foods such as marine fish and mammals that contain higher amounts of POPs than other foods $[24,44,50]$. In our study, Nenets had lower levels of p, $\mathrm{p}^{\prime}$-DDE and $\sum D D T$ than non-Nenets while the evidence for other pollutants was inconclusive in multivariable analysis. Thus, we may speculate that Nenets and non-Nenets from the same settlements may have similar food patterns and similar concentrations of POPs as suggested in other studies [26,30].

Age and BMI are other factors that need to be considered. We found positive associations between age, BMI and serum concentrations for half of the studied pollutants. Despite a global decreasing trend in PCBs and OCPs in the environment [51], the highest concentrations are often observed in the elderly due to higher environmental concentrations in the past and long biological half-lives of the POPs. $\Sigma$ DDT, p, p'-DDE and PCB 138, 153, 180 have the highest level of bioaccumulation in serum [37].

We emphasize that Arctic biomonitoring studies should consider the ethnic and geographic heterogeneity of the population as well as variations in food patterns and potential sources of POPs.

\section{Conclusions}

Serum concentrations of most PCBs and OCPs in the population of NAO are considerably lower than in other parts of the Arctic. Moreover, the levels of POPs in 2018 were substantially lower than in 2009. We observed significant geographic variations in serum concentrations of the PCB congeners 138, 153, 180, p, p'-DDE and HCB while ethnic variations were found only for $\mathrm{p}, \mathrm{p}^{\prime}$-DDE. These differences are likely to be attributed to the differences in nutrition. Our results demonstrate the need for biomonitoring studies for both men and women in Arctic Russia for the evaluation of long-term trends in legacy POPs and new emerging POPs. Further studies should concentrate on the factors behind the observed geographic and ethnic differences.

Author Contributions: This study has been developed with the contribution of all its authors. Conceptualization, Y.V., A.M.G., A.A. and T.S.; methodology, Y.V., A.A. and D.L.; validation, Y.V. and D.L.; formal analysis, Y.V., D.L., A.T., R.K., N.B., D.K. and A.M.G.; data curation, A.M.G. and Y.T.; writing—original draft preparation, Y.V., A.M.G., A.A. and T.S.; writing—review and editing, Y.V., 
D.L., A.A., A.T., R.K., D.K., N.B., L.P., A.M.G., V.C., T.S., J.Ø.O. and Y.T.; project administration, T.S. All authors have read and agreed to the published version of the manuscript.

Funding: The research was supported by the Government of the Russian Federation in compliance the Resolution of 9 April 2010 No. 220 (the contract from 14.03.2017 No. 14.Y26.31.0009). The authors declare that they have no financial or personal relationships that may have inappropriately influenced them in writing this article.

Institutional Review Board Statement: The study was conducted according to the guidelines of the Declaration of Helsinki and ap-proved by the by the Ethical Committee of Northern Medical State University, Arkhangelsk, Russia (protocol no. 06/09-17 of 27 September 2017).

Informed Consent Statement: Informed consent was obtained from all subjects involved in the study.

Data Availability Statement: The data presented in this study are available on request from the corresponding author.

Acknowledgments: Instrumentation of the Core Facility Center "Arktika" of Northern (Arctic) Federal University was used in this work. The work was carried out with the support of the world-class scientific and educational center "Russian Arctic: new materials, technologies and research methods".

Conflicts of Interest: The authors declare that there is no conflict of interest related to the publication of this article that should be reported.

\section{References}

1. Vested, A.; Giwercman, A.; Bonde, J.P.; Toft, G. Persistent organic pollutants and male reproductive health. Asian J. Androl. 2014, 16, 71-80. [CrossRef]

2. Wania, F.; MacKay, D. Peer Reviewed: Tracking the Distribution of Persistent Organic Pollutants. Environ. Sci. Technol. 1996, 30, 390A-396A. [CrossRef] [PubMed]

3. AMAP. AMAP Assessment 2009: Human Health in the Arctic; Arctic Monitoring and Assessment Programme (AMAP): Oslo, Norway, 2009; p. xiv + 256.

4. Dudarev, A.A. Persistent polychlorinated hydrocarbons and heavy metals in the Arctic biosphere: The main patterns of exposure and reproductive health of indigenous people. Biosphere 2009, 1, 186-202.

5. Tsygankov, V.Y.; Boyarova, M.D.; Donets, M.M.; Gumovskaya, Y.P.; Gumovskiy, A.N.; Koval, I.P.; Khristoforova, N.K.; Kiku, P.F.; Usov, V.V. Persistent organic pollutants (POPs) in the body of residents of the Russian Far East coastal regions. In Persistent Organic Pollutants (POPs) in the Far Eastern Region: Seas. Organisms. Human: Monograph; Tsygankov, V.Y., Donets, M.M., Khristoforova, N.K., Gumovskaya, Y.P., Polevshchikov, A.V., Gumovsky, A.N., Boyarova, M.D., Chernyaev, A.P., Lyagusha, M.S., Busarova, O., Eds.; Publishing House of the Far Eastern Federal University: Vladivostok, Russia, 2020; pp. 317-338.

6. AMAP. Biological Effects of Contaminants on Arctic Wildlife and Fish; Arctic Monitoring and Assessment Programme (AMAP): Tromsø, Norway, 2018.

7. Bravo, N.; Grimalt, J.O.; Chashchin, M.; Chashchin, V.P.; Odland, J.Ø. Drivers of maternal accumulation of organohalogen pollutants in Arctic areas (Chukotka, Russia) and 4,4'-DDT effects on the newborns. Environ. Int. 2019, 124, 541-552. [CrossRef] [PubMed]

8. Varakina, Y.; Lahmanov, D.; Aksenov, A.; Trofimova, A.; Korobitsyna, R.; Belova, N.; Sobolev, N.; Kotsur, D.; Sorokina, T.; Grjibovski, A.M.; et al. Concentrations of Persistent Organic Pollutants in Women's Serum in the European Arctic Russia. Toxics 2021, 9, 6. [CrossRef]

9. Stockholm Convention on Persistent Organic Pollutants Was Adopted on 22 May 2001 in Stockholm. Sweden. Available online: http:/ /chm.pops.int/Portals/0/Repository/convention_text/UNEP-POPS-COP-CONVTEXT-FULL.English.PDF (accessed on 19 May 2021).

10. Laird, B.D.; Goncharov, A.B.; Chan, H.M. Body burden of metals and persistent organic pollutants among Inuit in the Canadian Arctic. Environ. Int. 2013, 59, 33-40. [CrossRef]

11. Health Canada. Results of the Canadian Health Measures Survey Cycle 1 (2007-2009); Canadian Health Measures Survey: Ottawa, Canada, 2010; ISBN 78-1-100-15618-7.

12. Deutch, B. Recent dietary studies in the Arctic AMAP 2003; ch 7: 75-87. Available online: https://www.amap.no/documents/ download/181/inline (accessed on 1 December 2021).

13. Glynn, A.W.; Wolk, A.; Aune, M.; Atuma, S.; Zettermark, S.; Mæhle-Schmid, M.; Darnerud, P.O.; Becker, W.; Vessby, B.; Adami, H.-O. Serum concentrations of organochlorines in men: A search for markers of exposure. Sci. Total Environ. 2000, 263, 197-208. [CrossRef]

14. Zani, C.; Magoni, M.; Speziani, F.; Leonardi, L.; Orizio, G.; Scarcella, C.; Gaia, A.; Donato, F. Polychlorinated biphenyl serum levels, thyroid hormones and endocrine and metabolic diseases in people living in a highly polluted area in North Italy: A population-based study. Heliyon 2019, 5, e01870. [CrossRef] 
15. Sala, M.; Sunyer, J.; Otero, R.; Santiago-Silva, M.; Camps, C.; Grimalt, J. Organochlorine in the serum of inhabitants living near an electrochemical factory. Occup. Environ. Med. 1999, 56, 152-158. [CrossRef]

16. Popova, E.K.; Arkhipova, N.S.; Popov, I.O. Risk predictors of coronary heart disease in men of older age groups living in the conditions of far north. Hum. Ecol. 2020, 2, 4-11. [CrossRef]

17. Svetlichnaya, T.G.; Vorobyeva, N.A. Lifestyle and Self-Percieved health of the Nenets population living on the arctic island of vaigach. Hum. Ecol. 2019, 12, 20-25. [CrossRef]

18. Gumovskaya, Y.P.; Gumovskiy, A.N.; Tsygankov, V.; Polevshchikov, A.V. Persistent organic pollutants (POPs) in the human body: The experience of Russia and the ex-USSR republics. In Persistent Organic Pollutants (POPs) in the Far Eastern Region: Seas. Organisms. Human: Monograph; Tsygankov, V.Y., Donets, M.M., Khristoforova, N.K., Gumovskaya, Y.P., Polevshchikov, A.V., Gumovsky, A.N., Boyarova, M.D., Chernyaev, A.P., Lyagusha, M.S., Busarova, O.Y., et al., Eds.; Publishing House of the Far Eastern Federal University: Vladivostok, Russia, 2020; pp. 283-316.

19. Moshansky, V.F.; Kagan, S.A.; Tektinsky, O.L. Differential diagnosis of two forms of necrospermia. Urol. Nephrol. 1987, 4, 57-59.

20. Gromenko, D.S.; Galimov, S.N.; Shemagonov, D.V.; Farkhutdinov, R.R. The role of reactive oxygen species in the formation of male infertility. Kazan Med. J. 2007, 88, 23-24.

21. Murugesan, P.; Senthilkumar, J.; Balasubramanian, K.; Aruldhas, M.M.; Arunakaran, J. Impact of polychlorinated biphenyl Aroclor 1254 on testicular antioxidant system in adult rats. Hum. Exp. Toxicol. 2005, 24, 61-66. [CrossRef] [PubMed]

22. Khurtsilava, O.G.; Chashchin, V.P.; Meltser, A.V.; Dardynskaia, I.V.; Erastova, N.V.; Chashchin, M.V.; Dardynskiy, O.A.; Bazilevskaya, E.M.; Belikova, T.M.; Kovshov, A.A.; et al. Pollution of the environment with persistent toxic substances and prevention of their harmful impact on the health of the indigenous population residing in the arctic zone of the Russian federation Hyg. Sanit. 2017, 96, 409-414. [CrossRef]

23. Sorokina, T.Y. A national system of biological monitoring in the Russian Arctic as a tool for the implementation of the Stockholm Convention. Int. Environ. Agreem. Politi. Law Econ. 2019, 19, 341-355. [CrossRef]

24. Lakhmanov, D.; Varakina, Y.; Aksenov, A.; Sorokina, T.; Sobolev, N.; Kotsur, D.; Plakhina, E.; Chashchin, V.; Thomassen, Y Persistent Organic Pollutants (POPs) in Fish Consumed by the Indigenous Peoples from Nenets Autonomous Okrug. Environments 2019, 7, 3. [CrossRef]

25. Sobolev, N.; Ellingsen, D.G.; Belova, N.; Aksenov, A.; Sorokina, T.; Trofimova, A.; Varakina, Y.; Kotsur, D.; Grjibovski, A.M.; Chashchin, V.; et al. Essential and non-essential elements in biological samples of inhabitants residing in Nenets Autonomous Okrug of the Russian Arctic. Environ. Int. 2021, 152, 106510. [CrossRef]

26. Sorokina, T.; Sobolev, N.; Ellingsen, D.G.; Aksenov, A.; Trofimova, A.; Varakina, Y.; Kotsur, D.; Gribovski, A.M.; Chashchin, V.; Thomassen, Y. Essential and non-essential elements in whole blood and their association with diet and iron status: Results from an indigenous population study in Nenets Autonomous Okrug of the Russian Arctic. Environ. Int. 2021, submitted.

27. Bernert, J.T.; Turner, W.E.; Patterson, D.G., Jr.; Needham, L.L. Calculation of serum "total lipid" concentrations for the adjustment of persistent organohalogen toxicant measurements in human samples. Chemosphere 2007, 68, 824-831. [CrossRef]

28. Kim, S.; Park, J.; Kim, H.-J.; Lee, J.J.; Choi, G.; Choi, S.; Kim, S.; Kim, S.Y.; Moon, H.-B.; Kim, S.; et al. Association between Several Persistent Organic Pollutants and Thyroid Hormone Levels in Cord Blood Serum and Bloodspot of the Newborn Infants of Korea. PLoS ONE 2015, 10, e0125213. [CrossRef]

29. Dudarev, A.A.; Odland, J.O. Human health in connection with arctic pollution-results and perspectives of international studies under the aegis of AMAP. Hum. Ecol. 2017, 9, 3-14. [CrossRef]

30. Odland, J.; Deutch, B.; Hansen, J.C.; Burkow, I.C. The importance of diet on exposure to and effects of persistent organic pollutants on human health in the Arctic. Acta Paediatr. 2007, 92, 1255-1266. [CrossRef]

31. Kang, J.H.; Chang, Y.-S. Organochlorine Pesticides in Human Serum. In Pesticides-Strategies for Pesticides Analysis; Stoytcheva, M., Ed.; IntechOpen Publishing: Rijeka, Croatia, 2011; pp. 215-240.

32. Rylander, C.; Sandanger, T.M.; Petrenya, N.; Konoplev, A.; Bojko, E.; Odland, J.Ø. Indications of decreasing human PTS concentrations in North West Russia. Glob. Health Action 2011, 4, 8427. [CrossRef]

33. Long, M.; Wielsøe, M.; Bonefeld-Jørgensen, E. Time Trend of Persistent Organic Pollutants and Metals in Greenlandic Inuit during 1994-2015. Int. J. Environ. Res. Public Health 2021, 18, 2774. [CrossRef]

34. Porta, M.; Pumarega, J.; Henríquez-Hernández, L.A.; Gasull, M.; Bartoll, X.; Arrebola, J.P.; Morales, E.; Ibarluzea, J.; Alguacil, J.; Bilal, U.; et al. Reductions in blood concentrations of persistent organic pollutants in the general population of Barcelona from 2006 to 2016. Sci. Total. Environ. 2021, 777, 146013. [CrossRef]

35. Singh, K.; Chan, H.M. Association of blood polychlorinated biphenyls and cholesterol levels among Canadian Inuit. Environ. Res. 2018, 160, 298-305. [CrossRef] [PubMed]

36. Pavuk, M.; Olson, J.; Sjödin, A.; Wolff, P.; Turner, W.; Shelton, C.; Dutton, N.; Bartell, S. Serum concentrations of polychlorinated biphenyls (PCBs) in participants of the Anniston Community Health Survey. Sci. Total Environ. 2014, 473-474, 286-297. [CrossRef]

37. Thomas, G.O.; Wilkinson, M.; Hodson, S.; Jones, K.C. Organohalogen chemicals in human blood from the United Kingdom. Environ. Pollut. 2006, 141, 30-41. [CrossRef]

38. Zapevalov, M.A.; Samsonov, D.P.; Kochetkov, A.I.; Pasynkova, E.M.; Bogacheva, E.G. Global Atmospheric Transport of Persistent Organic Pollutants to the Russian Arctic. Russ. Meteorol. Hydrol. 2020, 45, 658-668. [CrossRef]

39. Novikov, M.A. Persistent organic pollutants in bottom sediments of the Barents Sea. Water Resour. 2021, 48, 334-343. [CrossRef] 
40. Long, M.; Knudsen, A.-K.S.; Pedersen, H.S.; Bonefeld-Jørgensen, E.C. Food intake and serum persistent organic pollutants in the Greenlandic pregnant women: The ACCEPT sub-study. Sci. Total Environ. 2015, 529, 198-212. [CrossRef] [PubMed]

41. D'Amato, C.; Torres, J.P.M.; Malm, O. DDT (dicloro difenil tricloroetano): Toxicidade e contaminação ambiental-uma revisão. Química Nova 2002, 25, 995-1002. [CrossRef]

42. Muir, D.; Savinova, T.; Savinov, V.; Alexeeva, L.; Potelov, V.; Svetochev, V. Bioaccumulation of PCBs and chlorinated pesticides in seals, fishes and invertebrates from the White Sea, Russia. Sci. Total Environ. 2003, 306, 111-131. [CrossRef]

43. Hop, H.; Borgå, K.; Gabrielsen, G.W.; Kleivane, L.; Skaare, J.U. Food Web Magnification of Persistent Organic Pollutants in Poikilotherms and Homeotherms from the Barents Sea. Environ. Sci. Technol. 2002, 36, 2589-2597. [CrossRef]

44. Polder, A.; Savinova, T.; Tkachev, A.; Løken, K.; Odland, J.Ø.; Skaare, J. Levels and patterns of Persistent Organic Pollutants (POPS) in selected food items from Northwest Russia (1998-2002) and implications for dietary exposure. Sci. Total Environ. 2010, 408, 5352-5361. [CrossRef] [PubMed]

45. Davydov, A.N. Climate change and living conditions in the Arctic as perceived by the Nenets of Vaygach Island. Ekologiya cheloveka Hum. Ecol. 2013, 2, 29-34. [CrossRef]

46. AMAP. Human Health in the Arctic; Arctic Monitoring and Assessment Programme (AMAP): Oslo, Norway, 2015; Available online: https:/ / www.amap.no/documents/doc/amap-assessment-2015-human-health-in-the-arctic/1346 (accessed on 7 June 2021).

47. Bjermo, H.; Darnerud, P.O.; Lignell, S.; Pearson, M.; Rantakokko, P.; Nälsén, C.; Barbieri, H.E.; Kiviranta, H.; Lindroos, A.K.; Glynn, A. Fish intake and breastfeeding time are associated with serum concentrations of organochlorines in a Swedish population. Environ. Int. 2013, 51, 88-96. [CrossRef] [PubMed]

48. Törnkvist, A.; Glynn, A.; Aune, M.; Darnerud, P.O.; Ankarberg, E.H. PCDD/F, PCB, PBDE, HBCD and chlorinated pesticides in a Swedish market basket from 2005-Levels and dietary intake estimations. Chemosphere 2011, 83, 193-199. [CrossRef]

49. Sobolev, N.; Nieboer, E.; Aksenov, A.; Sorokina, T.; Chashchin, V.; Ellingsen, D.G.; Varakina, Y.; Plakhina, E.; Kotsur, D.; Kosheleva, A.; et al. Concentration dataset for 4 essential and 5 non-essential elements in fish collected in Arctic and sub-Arctic territories of the Nenets Autonomous and Arkhangelsk regions of Russia. Data Brief 2019, 27, 104631. [CrossRef]

50. Savinov, V.; Muir, D.; Svetochev, V.; Svetocheva, O.; Belikov, S.; Boltunov, A.; Alekseeva, L.; Reiersen, L.-O.; Savinova, T. Persistent organic pollutants in ringed seals from the Russian Arctic. Sci. Total Environ. 2011, 409, 2734-2745. [CrossRef] [PubMed]

51. Abass, K.; Emelyanova, A.; Rautio, A. Temporal trends of contaminants in Arctic human populations. Environ. Sci. Pollut. Res. 2018, 25, 28834-28850. [CrossRef] [PubMed] 